\title{
Formação para Municípios Saudáveis: trajetória, experiência e desafios para a Rede Pernambucana de Municípios Saudáveis
}

DosSiER DE PESQUiSA : CidAde SAUdÁVEL

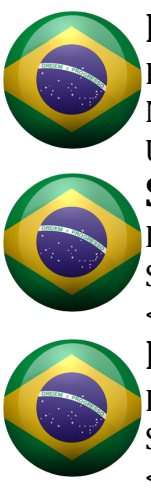

\section{Ronice Pereira Franco de Sá}

PhD em Educação, MSc em Administração de Serviços de Saúde, Médica Sanitarista, Pesquisadora do Núcleo de Saúde Pública e Desenvolvimento Social e Professora da Pós-Graduação em Saúde Coletiva da Universidade Federal de Pernambuco. Recife [PE] Brasil <ronicefranco@gmail.com>.

\section{Socorro Machado Freire}

Doutora em Saúde Pública, Mestre em Serviço Social, Assistente Social Sanitarista, Diretora do Núcleo de Saúde Pública e Desenvolvimento Social da Universidade Federal de Pernambuco. Recife [PE] Brasil $<$ socorromfreire@gmail.com>.

\section{Rosane de Senna Salles}

Doutoranda em Saúde Coletiva, Mestre em Antropologia, Médica Veterinária, Pesquisadora do Núcleo de Saúde Pública e Desenvolvimento Social da Universidade Federal de Pernambuco. Recife [PE] Brasil $<$ rosanesalles@terra.com.br>.

\section{Resumo}

0 artigo trata da trajetória da Rede Pernambucana de Municípios Saudáveis (RPMS), sua experiência e desafios específicos para a formação temática que realiza desde 2004, tendo como pano de fundo a reemergência do ideário das cidades saudáveis no contexto mundial, após o Encontro dos Prefeitos acontecido em Xangai, em 2016. São descritos os cursos principais, público-alvo e conteúdo programático. Reflexões teórico-conceituais são realizadas sobre a caminhada da RPMS. Em um novo cenário, deve-se considerar a agenda 2030 e os objetivos do desenvolvimento sustentável, um dos quais trata sobre o tema de municípios, cidades e comunidades saudáveis (MCCS). Apresenta-se o guia da Organização Panamericana de Saúde para a implementação da Declaração de Xangai e discute-se as possibilidades de adoção desse guia na nova formação proposta para a RPMS. Ressalta-se, ainda, os novos desafios que surgiram no âmbito da virtualização, da necessidade de mudar abordagem e conceito sobre promoção da saúde, da constatação do não-lugar e da mixofobia e aponta-se que esses temas emergentes precisam ser enfrentados na formação que a RPMS oferece.

\section{Palavras-chave}

Promoção da Saúde. Cidades Saudáveis. Sustentabilidade. Formação. Declaração de Xangai.

\section{Training for Healthy Municipalities: trajectory, experience and challenges for the "Pernambuco" Network of Healthy Municipalities}

\begin{abstract}
This paper deals with the Pernambuco Network of Healthy Municipalities (RPMS), its experience and specific challenges for thematic training that it has been doing since 2004, in the context of the reemergence of the ideal of healthy cities in the world context, after the Meeting of Mayors which took place in Shanghai in 2016. The main courses, target audience and program content are described. Theoretical-conceptual reflections are carried out on the RPMS journey. In a new scenario, one should consider the 2030 agenda and the goals of sustainable development, one of which addresses the issue of healthy cities, towns and communities (MCCS). It presents the guide of the Pan American Health Organization for the implementation of the Shanghai Declaration and discusses the possibilities of adopting this guide in the new training proposed for the RPMS. The new challenges that have arisen in the area of virtualization, the need to change approach and concept on health promotion, non-location and mixofobia, and it is pointed out that these emerging themes need to be addressed in the formation that the RPMS offers.
\end{abstract}

\section{Keywords}

Health Promotion. Healthy Cities. Sustainability. Training. Shanghai Declaration. 


\section{Introdução}

Em tempos de reemergência do ideario por Municípios, Cidades e Comunidades Saudáveis (MCCS) enquanto estratégia ou agenda política, faz-se necessário apresentar a experiência de formação específica para esta temática que a Rede Pernambucana de Municípios Saudáveis (RPMS) tem desenvolvido desde 2004, bem como os desafios para esta formação em virtude de mudanças de contexto e reflexões sobre o marco teórico-conceitual de interesse.

O ressurgimento das discussões sobre a estratégia de MCCS para a América Latina tem sido verificado desde 2016 com:

a. a Declaração de Santiago, fruto do Pré-Fórum de Prefeitos das Américas "Rumo a Xangai 2016";

b. a Declaração de Xangai, resultado da 9a Conferência Global de Promoção da Saúde;

c. o Consenso dos Prefeitos de Cidades Saudáveis de Xangai;

d. o aval da Rede Latino-Americana e do Caribe de Gestores de Promoção da Saúde (REDLACPROMSA);

e. o Encontro de Prefeitos da região das Américas: saúde em todas as políticas para implementar a Agenda 2030 - México 2018.

No Brasil, durante os últimos anos, a estratégia foi mantida por conta de universidades e outros centros formadores. Em sua maioria, estes entendiam a importância de manter o foco no conceito de território articulado com o de comunidade enquanto tarefa fundamental para analisar determinantes socioambientais, bem como para analisar processos saúde-doença e promover saúde no local em que se vive (Porto, Zancan, \& Pivetta, 2014).

Sem dúvida uma estratégia muito bem-sucedida na Europa (Wilding et al., 2017), o movimento por municípios, cidades e comunidades saudáveis passou por um período de muitos questionamentos e várias outras agendas mundiais com foco no território (cidade/município) surgiram, tais como: Cidades Justas, Cidades Verdes, Cidades Sustentáveis, Cidades Resilientes, Cidades Criativas, Cidades de Conhecimento, Cidades e Comunidades Seguras, Cidades Festivas, Cidades Inteligentes, Cidades Educadoras, Cidades Amigas das Crianças, Cidades Amigas dos Idosos, Cidades Inclusivas, Cidades Conscientes, dentre tantas outras. Segundo De Leeuw (2017) toda essa explosão de agendas, políticas, programas com o foco no território comprovam a importância dos assentamentos humanos para a mudança social, para a busca por oportunidades locais e globais no intuito de fazer do local em que se vive, o melhor lugar para seus habitantes e todos os outros usuários deste espaço.

Apesar da grande variedade de agendas apresentadas utilizarem o termo "cidades" em comum, registramos que o termo não apresenta unanimidade conceitual. Dessa forma, sempre que tratarmos de cidades saudáveis ou mesmo, de municípios, cidades e comunidades saudáveis (MCCS), termo adotado pela América Latina, estaremos nos referindo a assentamentos/povoamentos humanos. De acordo com Doxiadis (1970), criador do termo Eqüística, ou "Ekistics", ciência dos povoamentos humanos, esta ciência seria um sistema de cinco elementos: a natureza; o homem; a sociedade; as construções; as redes formadas por estes. A eqüística serviria para analisar os princípios que o homem considera ao criar seus povoamentos e a evolução destes em termos de tamanho e qualidade. Teria por meta, portanto, contribuir para a construção de uma cidade. Segundo Doxiadis (1970), os assentamentos humanos respondem a 5 princípios:

a. maximização dos contatos potenciais do homem com elementos da natureza, com outras pessoas e com aquilo que produz (como estradas e prédios);

b. minimização dos esforços necessários para conseguir contatos potenciais e efetivos;

c. otimização do espaço de proteção humano a fim de evitar desconforto sensorial e psicológico ao homem;

d. otimização da qualidade do relacionamento do homem com seu meio ambiente, que consiste na natureza, na sociedade e nas construções (este princípio leva ao ordenamento 
psicológico e estético que influencia a arquitetura e, em muitos aspectos, a arte);

e. capacidade da sociedade de organizar a si e a seus povoamentos, tentando obter uma síntese dos quatro primeiros princípios.

Ainda, de acordo com Doxiadis (1977) os humanos necessitam de cinco coisas nos seus assentamentos: liberdade para se mover, segurança, qualidade de vida que satisfaça as suas aspirações, contatos humanos e, criatividade e desenvolvimento humano.

\section{Rede Pernambucana de Municípios Saudáveis em poucas linhas}

Partindo das premissas apresentadas anteriormente, entendemos que a RPMS não se prende ao conceito de cidades ou municípios. Em alguns dos municípios da Rede as atividades são facilitadas, acompanhadas e executadas para e por agrupamentos específicos.

No entanto, nos cabe explicar que o projeto de cooperação internacional entre Brasil e Japão que deu origem à RPMS, iniciou-se com foco na promoção da saúde, em 2003. 0 que não era evidente era qual "promoção da saúde" seria a base para o projeto "Municípios Saudáveis no Nordeste do Brasil”.

A cooperação internacional era composta por peritos japoneses enviados pela Agência de Cooperação internacional do Japão (JICA), por pesquisadores e peritos do Núcleo de Saúde Pública e Desenvolvimento Social (NUSP) da Universidade Federal de Pernambuco (UFPE) e por técnicos da Secretaria de Planejamento e Gestão do governo do estado de Pernambuco. Mas, sendo Promoção da Saúde um termo polissêmico, com abordagem multiníveis, o grande desafio foi realizar um alinhamento conceitual para conduzir a atividade.

Os peritos japoneses estavam ancorados na abordagem comportamental, tinham seu foco no nível individual e apostavam num projeto sobre estilos de vida saudáveis e eram, eles mesmos, enquanto profissionais, um médico clínico, uma enfermeira hospitalar, uma especialista em Brasil e um administrador de projetos.

Aos técnicos do governo estadual cabia estabelecer a conexão do projeto e inseri-lo nas políticas públicas intersetoriais. No entanto, houve mais uma postura observadora, a princípio, uma vez que o projeto não demandava investimento direto de sua parte. Como esta instituição foi "convidada", acredita-se não ter havido o pertencimento necessário para uma ação conjunta desde o início. Afirmava-se que as outras instituições precisavam provar que haveria resultado ou impacto no prazo de uma gestão governamental. Mas, os poucos técnicos que abraçaram o projeto conseguiram influir na sustentabilidade deste no nível governamental (Franco de Sá et al., 2014; Freire, Franco de Sá, \& Gurgel, 2017).

À universidade coube iniciar uma série de discussões e reflexões que convergiam para a abordagem da Promoção da Saúde multidimensional. Os pesquisadores do NUSP apostavam na transformação social e na capacitação das populações dos municípios/assentamentos participantes. Assim, nasceu o modelo estruturador da RPMS com atuações de nível macro, meso e micro. No nível micro trabalha-se mais com o método Bambu (Menezes, Franco de Sá \& Freire, 2006) desenvolvido pelos integrantes do Projeto, mas que pode ser adotado também nos outros níveis.

Na ocasião, em 2003, o Projeto adotava o conceito que o Grupo Temático (GT) em Promoção da Saúde (PS) e Desenvolvimento Local Integrado e Sustentável (DLIS) -hoje chamado GT em PS e Desenvolvimento Sustentável (GTPSDS) da Associação Brasileira de Saúde Coletiva (ABRASCO) havia elaborado e que caracterizava a PS em 3 níveis: estilos de vida, gestão intersetorial e modelo de desenvolvimento.

Seguindo essa linha de pensamento, foi proposta uma formação específica para promotores de municípios saudáveis que começou experimentalmente em 2004 e foi retomada em 2007 (Franco de Sá et al., 2008) com apoio, em 2007, da JICA e nos dois anos seguintes pelo Ministério da Saúde, dentro da proposta de centros colaboradores em Promoção da Saúde para aquele ministério. 
Muitos foram os caminhos percorridos na busca da melhor abordagem mediante parcerias institucionais, participação em grupos de pesquisa, parcerias internacionais. Desde 2003, no entanto, o foco da RPMS era a busca do bem-estar e, por isso, a sua obsessão em elaborar um método que colocava a felicidade e a satisfação (dos desejos mais que das necessidades) no centro de suas ações, ao mesmo tempo tentando conectá-la com a redução das iniquidades socioculturais e regionais que assolam o nosso país e a região nordeste, em particular.

Dentre os grandes encontros e parcerias que fortaleceram as perspectivas iniciais e nos apresentaram outras e com os quais aprendemos e revimos a nossa forma de construir saber e de trabalhar no contexto dos MCCS, a Rede de Municípios Potencialmente Saudáveis (RMPS) foi, sem dúvida, uma grande fonte de trocas, aprendizados e intercâmbios. Pouca participação direta ou parceria tivemos com outras entidades colaboradoras responsáveis pela temática no Brasil. Registramos a participação em uma pesquisa com a Faculdade de Saúde Pública da Universidade de São Paulo (FSP/USP) - pesquisa multicêntrica para as 5 regiões do Brasil e que visava analisar o papel das agendas Cidades Saudáveis, Desenvolvimento Local Integrado e Sustentável (DLIS) e Agenda 21 nos Objetivos do Milênio (ODM).

0 percurso da RPMS e a sua ligação com a RMPS passou ainda por um projeto de cooperação internacional junto com a Associação Canadense de Saúde Pública (ACSP), a ABRASCO e a FIOCRUZ (ABRASCO, 2010). Esse projeto, denominado Ações Intersetoriais em Promoção da Saúde (AIPS) contava com outros pontos focais, além das duas redes citadas: reorientação de Agentes Comunitários de Saúde nas práticas de Atenção Primária em Saúde (Goiânia, GO), Ambientes Saudáveis (Curitiba, PR), DLIS em Manguinhos (Rio de Janeiro, RJ), Estratégia de Saúde da Família em interface com as políticas públicas municipais (Sobral, CE). No caso da RPMS, o trabalho com a cooperação canadense foi realizado junto às mulheres artesãs de Barra de Guabiraba, PE (Franco de Sá, Freire \& Senna Salles, 2010).

Com a RMPS tivemos vários outros momentos e discussões e muito usamos a vasta bibliografia publicada (Sperandio, 2003; Sperandio, 2004a; Sperandio 2004b; Sperandio \& Vilarta, 2004; Sperandio \& Serrano, 2006; dentre outras) que discutia desde mobilização, participação social, papel das universidades, sustentabilidade até a apresentação do Plano Diretor como ferramenta para desenvolvimento de políticas públicas em um município potencialmente saudável. Em Pernambuco, também trabalhamos com a elaboração de dois Planos Diretores Saudáveis para os municípios de Bonito e de Sairé.

\section{Formação em Promotores de Municípios Saudáveis}

A Rede Pernambucana de Municípios Saudáveis (RPMS) é constituída por 24 municípios inseridos em todas as regiões do estado de Pernambuco. Desde 2007 iniciou uma formação específica (após a formação experimental de 2004) para voluntários dos cinco primeiros municípios que faziam parte do projeto de cooperação internacional Brasil-Japão. Naquele ano, aconteceu o Módulo Zero, realizado em Camocim de São Félix, com o objetivo de promover interação entre as equipes locais do Projeto Municípios Saudáveis Nordeste do Brasil e entre os municípios.

Neste encontro, além da primeira capacitação sobre os pressupostos teóricos, incluindo pilares e valores da Promoção da Saúde e do ideário de Cidades Saudáveis e a proposta metodológica do Método Bambu (participação social, intersetorialidade, equidade, solidariedade, diversidade, voluntariado, empoderamento), também foram escolhidos e eleitos pelos seus pares, os supervisores (representantes da gestão) e os facilitadores (representantes da comunidade) responsáveis pelo desenvolvimento das ações locais de municípios saudáveis.

Esta ação proporcionou trocas de informação entre os municípios sobre suas potencialidades e identidade local, dando início à formação de vínculos na construção da "redinha" (o embrião da RPMS), como era chamada pelos Promotores de Municípios Saudáveis, a interação entre os cinco municípios piloto.

O Módulo Zero trouxe como um dos resultados principais o reconhecimento das potencialidades, identificação e legitimação de lideranças locais. E como consequência de um processo integrado 
e participativo, por sua vez, fez emergir o sentimento de pertencimento e identidade local, começando a mobilizar as pessoas e comunidades a descobrir os seus espaços e funções na intervenção, tomando inclusive a defesa do projeto para seus municípios, quando alguns prefeitos quiseram recuar, ao ficarem cientes de que a proposta não traria investimentos financeiros para as prefeituras, e as lideranças publicamente afirmaram como resposta: "estão trazendo conhecimento e é o que queremos para os nossos municípios". Com esta atitude, os representantes municipais convenceram os gestores a continuarem com o Projeto Municípios Saudáveis.

Os cursos de Formação de Promotores de Municípios Saudáveis, incluindo os seminários, foram constituídos enquanto eixos estruturadores da ação. Os primeiros cursos ocorreram em várias versões nos anos de 2007, 2008, 2009 e 2013 constituindo um critério para inclusão dos municípios na Rede Pernambucana de Municípios. No decorrer desses anos de cursos de promotores de municípios saudáveis foram realizados 7 (sete) seminários e foram formados 600 promotores de municípios saudáveis.

A primeira versão dessa formação contou com a cooperação de professores e especialistas que compunham o GT PSDS da ABRASCO. Contou também com apoio de especialista canadense em Desenvolvimento Local, além de parceiros locais da Secretaria Estadual de Saúde.

A formação foi oferecida para os municípios pernambucanos de Barra de Guabiraba, Bonito, Camocim de São Félix, Sairé e São Joaquim do Monte, e tinha como temas fixos:
a. Conceitos de Municípios Saudáveis e de Promoção da Saúde
b. Participação Social
c. Método Bambu
d. Empoderamento
e. Capital Social
f. Avaliação Participativa
g. Políticas Públicas Saudáveis
h. Desenvolvimento Local
i. Associativismo e Cooperativismo
j. Elaboração de Planos de Municípios Saudáveis (trabalho de conclusão de curso)

A segunda e terceira versões do curso ocorreram nos anos de 2008 e 2009 em 5 (cinco turmas), segundo critério de aglomerado territorial e secretarias estaduais. Teve carga horária geral de 180 horas em 10 módulos distribuídos em período de 9 (nove) meses. A terceira versão da formação de promotores ocorreu no ano de 2009 em 4 (quatro) módulos distribuídos em 3 (três) turmas segundo critérios de aglomerado territorial e secretarias estaduais, tendo carga horária total de 32 horas. No ano de 2013 uma versão condensada do curso foi ofertada em 3 módulos com carga horária total de 24 horas para novos municípios integrantes da Rede Pernambucana de Municípios Saudáveis. O público-alvo é formado pelos gestores locais, os profissionais de saúde, de educação, da área social, outros que tenham interesse e membros da sociedade civil que queiram ser voluntários promovendo municípios saudáveis.

A partir de 2009, durante 5 anos, este curso passou a ser oferecido internacionalmente para países da América latina e países africanos de língua oficial portuguesa (PALOPs). Isso acontecia dentro do programa Third Countries Training Program (TCTP) da JICA. Alunos do mestrado em Saúde Coletiva da UFPE também podiam participar do curso. Teve como objetivo formar Promotores de Municípios Saudáveis nos países convidados: Angola, Cabo Verde, Guiné Bissau, Moçambique, São Tomé e Príncipe, (membros da África PALOPs) e, Bolívia, Chile, Equador, El Salvador, Colômbia, Guatemala, Honduras, México, Nicarágua, Panamá, Paraguai, Peru, República Dominicana, Uruguai e Venezuela. A partir do modelo construído no Projeto Municípios Saudável no Nordeste do Brasil, que teve como principal resultado a formação e difusão da Rede Pernambucana de Municípios Saudáveis, essa experiência foi a base para o desenvolvimento de metas a realização de cinco edições do Curso internacional de Treinamento para a Promoção da Saúde, Desenvolvimento Local e Municípios Saudáveis. Aconteceram também missões conjuntas para 
monitoramento nos países participantes e a realização, em 2012, do I Encontro Internacional de Municípios Saudáveis da América Latina e PALOPs.

Na experiência dos processos de formação de RPMS, os cursos e seminários potencializaram um espaço de interação entre sujeitos (representantes da gestão e da comunidade) que ocupavam um lugar em uma comunidade, município, e território em que se colocavam como atores de uma ação coletiva a ser desenvolvida para atender a um objetivo comum, uma vez que, ao longo do curso, todos os alunos elaboravam um plano de ação para ser executado localmente. A construção destes planos possibilitou ação reflexiva sobre os contextos, competências e habilidade em promoção da saúde. Estas características da formação contribuíram para que se tornasse uma experiência inovadora/transformadora. 0 que, segundo Potvin e Clavier (2013), seria um espaço de prática de tradução por atender, tornar possível os dispositivos para viabilizar a tão almejada intersetorialidade, de que se pressupõe como pilares da promoção da saúde e municípios saudáveis. E foi constatado por Freire, Franco de Sá e Gurgel (2017) que a formação é uma das categorias responsáveis pela sustentabilidade da RPMS aliada ao reconhecimento do que já havia sido construído e à liderança estratégica.

\section{Novos desafios para pensar a formação para MCCS}

Desde o início dessa trajetória até o momento, muitas mudanças surgiram nos marcos teóricoconceituais adotados. Hoje, concordamos com a necessidade da conexão urgente entre os campos de planejamento urbano e de promoção da saúde. Faz-se necessário promover saúde urbana com equidade. Segundo Corburn (2017) o século 21 será o século das cidades e, em torno de 2050, 70\% da população mundial viverá em áreas metropolitanas. Além do mais, observa-se que os desafios para se alcançar saúde urbana com equidade são imensos, haja vista a disseminação global de doenças, a poluição ambiental além-fronteiras, o surgimento cada vez maior de favelas, o aumento das disparidades e iniquidades entre classes sociais, gênero e raça/etnia. Corburn ressalta que, apesar da reconexão entre planejamento e saúde pública ser imperativa deve-se monitorar e avaliar cada proposta que surgir considerando contextos político e cultural (especialmente) e perspectiva histórica.

Assim, hoje, acreditamos que sempre nos mantivemos guiados pelo conceito de promoção da saúde emancipatória: [...]

[...] processo dinâmico de mediações constituídas de campos relacionais, cognitivos e éticos, entre sujeitos individuais e coletivos para solidariamente estabelecerem mecanismos de compartilhamento dos recursos disponíveis na sociedade. Uma promoção da saúde repensada enquanto processo dialético voltado à produção de conhecimentos e práticas que favoreçam a constituição de espaços de conquistas de liberdade, de redução de vulnerabilidades socioambientais e de exercício dos direitos humanos fundamentais [...] (Porto \& Pivetta, 2009 citados por Porto, Zancan, \&Pivetta, 2014).

Na nossa caminhada, enquanto representantes da universidade na RPMS, sempre focamos na questão emancipatória. Por essa razão sempre cuidamos do lugar que deve ser dado aos valores e princípios e que são o fundamento das relações que os indivíduos estabelecem consigo mesmos e entre todos. A abordagem multidimensional (relacional, cognitiva e ética) é compatível com nosso modelo estruturador que expõe a necessidade de ação nos níveis micro, meso e macro.

Enfatizamos valores como felicidade, criatividade, amorosidade, demos espaço para explicitação de desejos, tendo como alvo a redução de iniquidades, a justiça social, a democracia, a solidariedade, a prática reflexiva, ações afirmativas e arte, muita arte (Rice et al., 2017, p. 197). 0 método Bambu mostrou-se flexível (daí a obviedade do seu nome) para transitar do campo dos valores individuais, aos relacionais indo até à elaboração de planos que visavam melhorias estruturais nos assentamentos/povoamentos envolvidos.

O foco nos valores está destacado em uma dentre as seis orientações políticas transformadoras propostas pela Organização Pan-Americana de Saúde (OPAS, 2018) no seu Guia para a implementação nacional da Declaração de Xangai. A orientação política "sociedades produtivas para o 
bem-estar" está na mesma linha de orientação que a RPMS tem adotado desde 2003 com publicações acerca do tema desde 2006 (Melo Filho, Franco de Sá, \& Chuma, 2007; Franco de Sá et al., 2007; Franco de Sá et al., 2006) e também com participação como informantes-chave em pesquisas realizadas pelos pesquisadores e consultores da OPAS. Com grande satisfação que a equipe acadêmica da RPMS recebeu a notícia que a Organização para a Cooperação e Desenvolvimento Econômico (OCDE), em junho de 2016, se comprometeu a "redefinir a narrativa do crescimento de modo a colocar o bem-estar das pessoas no centro dos esforços dos governos" (OPAS, 2018, p. 12). O Relatório Mundial de Felicidade (World Happiness Report), publicado pela Sustainable Development Solutions Network defende que para haver felicidade é preciso observar a interface entre fatores econômicos e sociais e classifica os países segundo seis categorias de indicadores: liberdade, generosidade, apoio social, saúde, renda e governança confiável.

Se, à época da pesquisa multicêntrica citada (com a FSP/USP) tínhamos a agenda 21 e os Objetivos do Milênio (ODM) para dialogar com a agenda de Cidades saudáveis, hoje precisamos colocar foco na Agenda 2030 e seus Objetivos do Desenvolvimento Sustentável (ODS) para realinhar seja a estratégia por MCCS e, até mesmo, os conceitos já validados de promoção da Saúde.

O Guia para implementação nacional da Declaração de Xangai aponta caminhos que precisam ser adotados para atualizar as formações tanto em Municípios quanto em promoção da Saúde. 0 Guia analisa o contexto atual apontando três pressupostos básicos: a interconexão do mundo atual tem repercussões na forma de pensar, promover e fazer saúde; a saúde do planeta está em risco e precisamos investir no desenvolvimento sustentável; e, a liderança política arrojada é uma necessidade essencial para se garantir o bem-estar, a felicidade e a sustentabilidade para as populações e o planeta(OPAS, 2018).

O modelo transformador, proposto pela OPAS (e pela OMS) está assim expresso:

A) Orientações políticas transformadoras.

a. Sociedades produtivas para o bem-estar - foco no bem-estar das pessoas, na felicidade, na justiça social;

b. Interconexão - buscar conectar agendas, políticas, programas e projetos;

c. "Não deixar ninguém para trás" - análise de um caminho crítico entre países avaliando aqueles com maior e menor progresso no alcance das metas da Agenda 2030;

d. Abordagens criadoras de valor para o desenvolvimento econômico - como exemplo, ciclismo, cria valor sobre as interferências climáticas e ajuda a prevenir doenças cardíacas, alguns cânceres, diabetes tipo 2, riscos sobre a obesidade, menos poluição, etc.;

e. Transformação verde do setor da saúde - existência de hospitais verdes e saudáveis que levam em consideração resiliência climática, liderança, governança, etc.;

f. Gênero e outras relações sociais - as diferenças de gênero são grandes quando analisamos morbidade e mortalidade. Investir mais no empoderamento de mulheres e meninas ajuda a gerar equidade no mundo.

B) Criação de vontade política para a ação.

a. Transformação por meio dos mais altos níveis de ação política - por exemplo: o Uruguai desafiou a indústria do tabaco e venceu;

b. Ênfase forte nas questões locais - cidades como plataforma virtual MCCS;

c. Ênfase nas pessoas - centralidade do letramento em saúde;

d. Transformação por meio de governança participativa;

e. Fortalecimento da governança global da saúde por meio da coerência entre as políticas;

f. Transformação dos ministérios da saúde.

C) Possibilitar capacidades de governança transformadoras nos países.

a. Abordagens para a Saúde em Todas as Políticas (Health In All Policies - HIAP);

b. Fortalecimento da legislação, regulamentação e tributação de produtos não saudáveis;

(C) Labor \& Engenho, Campinas [SP] Brasil, v.12, n.4, p.509-518, out./dez. 2018. 
c. Conhecimento e transformação de dados;

d. Transformação tecnológica e científica - virtualização das relações, do convívio, do trabalho, do viver;

e. Fortalecimento dos sistemas de saúde pública e organização da promoção da saúde;

f. Abordagem de determinantes comerciais da saúde - combate aos interesses comerciais prejudiciais à saúde. Os principais vilões são as indústrias de alimentos processados, de bebidas alcóolicas e do tabaco;

g. Parcerias e abordagens participativas;

h. Capacitação para novas formas de trabalho.

Focar em assentamentos/povoametos como propunha Doxiadis pode, erroneamente, aparentar um monolito humano, um campo de pertencimento, solidariedade e alteridade. É por isso que a tradição antropológica ligou a questão da alteridade (ou da identidade) à do espaço porque os processos de simbolização colocados em prática pelos grupos sociais deveriam compreender e controlar o espaço para se compreenderem e se organizarem a si mesmos (Augé, 2005). Os três tipos de alteridade: a) social (diferença de sexo, idade, classe social etc.), b) íntima (aquilo que cada um consegue fazer de si próprio) e c) completa (o estrangeiro, entendido como o inimigo) sempre existiram na história das sociedades. Mas o que se passa hoje, no período que Augé (2005) denominou sobremodernidade - marcado pelos excessos de acontecimentos, imagens e referências espaciais e individuais - é a perda da categoria do outro.

O que temos quando perdemos a categoria do outro são os "não lugares". Estes, permitem uma grande circulação de pessoas, coisas e imagens em um único espaço, transformando o mundo em um espetáculo com o qual mantemos relações a partir das imagens, transformando-nos em espectadores de um lugar profundamente codificado, do qual ninguém faz verdadeiramente parte (Augé, 2005). Nesse não-lugar trocamos (Franco de Sá, 2018):
a. Pessoas por algoritmos;
b. Fatos por Fakenews;
c. Pesquisas científicas por "dar um Google";
d. Rodas de conversas por WhatsApp.

Como falar em assentamentos humanos, municípios ou cidades quando a identidade/pertencimento, hoje, está sujeita a algoritmos que direcionam mensagens que são visualizadas em telas individualizadas? Temos uma "Sociedade" digital ou um Monolito humano digital? Está em nossas mãos viver vidas que não são as nossas, não considerar os fatos e transformar os lugares em não lugares ? (Franco de Sá, 2018).

A perda da categoria do outro é também ditada pelo "medo que assola as cidades com a perda da solidariedade coletiva" (Akerman, Mendes, \& Fischer, 2014, p.77). Os autores citados demonstram espanto ao verificar que esse desafio apontado por pesquisadores que discutem cidades não é tratado pelos ativistas do campo da promoção da saúde. Para eles, citando Bauman (2009), esse medo não é somente consequência da insegurança urbana mas constitui-se num efeito da mixofobia - o medo de misturar-se. Segundo Bauman (2009, citado por Akerman, Mendes, \& Fischer, 2014): [...]

[...] essa mixofobia não passa da difusa e muito previsível reação a impressionante e exasperada variedade de tipos humanos e de estilos de vida que se podem encontrar nas ruas da cidade [...] as tensões derivadas da "estrangeiridade" incômoda e desorientadora desse cenário acabarão, provavelmente por favorecer as tendências segregacionistas (Bauman, 2009).

Nova formação está sendo proposta para 2019 e, entendemos que os temas expostos enquanto desafio precisam ser abordados e, antes disso, precisam ser aprofundados e debatidos nos fóruns existentes, correndo o risco de termos uma RPMS anacrônica e superficial. Os gestores precisam enfrentar a necessidade de promover coerência entre as políticas, de ampliar a aborda- 
gem sobre saúde, de adotar a agenda 2030 e a multidimensionalidade da estratégia MCCS. Atuar desde o campo dos valores, da cognição, da ética e enfrentar os desafios econômicos, culturais e sociais, como os determinantes comerciais da saúde, a sociedade digital e, paradoxalmente, a emergência do não-lugar e da perda de alteridade, sinais desses tempos virtuais onde reemerge a necessidade do território físico.

\section{Referências}

Abrasco (2010). A decade of Canadian and Brazilian Cooperation in Public Health. Knowledge exchange and learning to strengthen intersectoral health promotion. Recuperado de: <http://www.abrasco.org.br/ publicacoes/arquivos/20100707183931.pdfntercs>

Akerman, M., Mendes, R., \& Fischer, A.L. (2014). Cidades Saudáveis: ainda um tema relevante? In C. B. Silveira, T. M. Fernandes. \& B. Pellegrini (orgs.). Cidades Saudáveis? Alguns olhares sobre o tema (pp. 269-299). Rio de Janeiro: Editora Fiocruz.

Augé, M. (2005). Não lugares: introdução a uma antropologia da sobremodernidade. Lisboa: 90 Graus.

Corburn, J. (2017). Equitable and Healthy City Planning: Towards Healthy Urban Governance in the Century of the City. In E. De Leeuw \& J. Simos (orgs.). Healthy Cities - The Theory, Policy, and Practice of Value-Based Urban Planning (pp. 31-41). New York: Springer.

De Leeuw, E. (2017). Cities and Health from the Neolithic to the Anthropocene. In E. De Leeuw \& J. Simos (orgs.). Healthy Cities - The Theory, Policy, and Practice of Value-Based Urban Planning (pp.3-30). New York: Springer.

Doxiadis, C.A. (1970). Ekistics, the science of the human settlements. Science, 170 (3956), pp. 393-404.

Doxiadis (1977). Ecology and ekistics. Boulder: Westview Press.

Franco de Sá, R., Salles, R., Freire, S. \& Schmaller, V. (2014). A Teoria Ator-Rede e a compreensão do processo de sustentabilidade das intervenções em Promoção da Saúde: contributos e lições aprendidas. In Z. Hartz, L. Potvin \& R. Bodestain. (orgs). Avaliação em Promoção da Saúde (pp.201-211). Brasília: CONASS.

Franco de Sá, R., Freire, M. S. M., Yamamoto, S., \& Senna Salles, R. P. (2008). Caderno de Formação de Promotores de Municípios Saudáveis. Recife: Editora Universitária UFPE.

Franco de Sá, R. (2018). Construindo cidades na perspectiva dos ODS: inclusão, segurança, resiliência e sustentabilidade. In 6a Conferência Regional Latinoamericana de Promoção da Saúde e Educação em Saúde da UIPES/ORLA. Franca, SP, Brasil, 8-10 de outubro de 2018. Recuperado de: <http:// www.oxfordeventos.com.br/promocaodasaude2018/>.

Franco de Sá, R., Araújo, J. A., Freire, M. S. M., Senna Salles, R., Chuma, J., Royama, H., Yuasa, M., Yamamoto, S., Menezes Filho, A., Nishida, M., Trindade, C. M. A., \& Oliveira, A. A. (2007). Manual do Método Bambu construindo municípios saudáveis. Recife: Ed. Universitária UFPE.

Franco de Sá, R., Viana, V., Nishida, M., \& Yuasa, M. (2006). UFPE/NUSP/Recife: municípios saudáveis no Nordeste do Brasil. In M. Akerman \& R. Mendes (orgs.). Avaliação Participativa de Municípios, Comunidades e Ambientes Saudáveis: a trajetória brasileira - memória, reflexões e experiências. São Paulo: Mídia Alternativa.

Franco de Sá, R., Freire, S., Senna Salles, R. (2010). Barra de Guabiraba/PE. In H. Monteiro, J. Chauvin, A.I. Carvalho, C.S. Silva \& A.H. Matida (orgs.). Ações Intersetoriais em Promoção da Saúde. Rio de Janeiro: ABRASCO.

Freire, S., Franco de Sá, R., \& Gurgel, I. G. D. (2017). Sairé Mais Saudável: política intersetorial como marco de mudança para equidade local. Ciênc. saúde coletiva, 22(12). Recuperado de: <http://www.scielo.br/ scielo.php?script=sci_arttext\&pid=S1413-81232017021203893>. 
Melo Filho, D. A., Franco de Sá, R., \& Chuma, J. (2007). Avaliação do capital social nas áreas de atuação do projeto Municípios Saudáveis no Nordeste do Brasil - Barra de Guabiraba-Bonito - Camocim de São Félix Sairé - São Joaquim do Monte. Recife: Edições Bagaço.

Menezes, A., Franco de Sá, R., \& Freire, S. (2006). Método Bambu. In R. Franco de Sá, M. Yuasa, \& V. P. Viana (orgs.). Municípios Saudáveis no Nordeste do Brasil. Conceitos, Metodologia e Relações Institucionais (pp. 49-58). Recife: Editora Universitária UFPE.

OPAS. Organização Pan-Americana de Saúde. (2018). Promoção da Saúde: Guia para a implementação nacional da Declaração de Xangai. Brasília: OPAS/BRA/OMS.

Porto, M. F., Zancan, L., \& Pivetta, F. (2014). Cidades Saudáveis e Promoção da Saúde Emancipatória: reinvenção cotidiana do (re)conhecimento nos territórios vulneráveis. In C. B. Silveira, T. M. Fernandes, \& B. Pellegrini (orgs.). Cidades Saudáveis? Alguns olhares sobre o tema (pp. 269-299). Rio de Janeiro: Editora Fiocruz.

Potvin, L. \& Clavier, C. (2013). Actor-Network Theory: The governance of intersectoral initiatives. In C. Clavier \& E. De Leeuw (eds.). Health Promotion and the Policy Process (pp. 82-103). Oxford: Oxford University Press.

Rice, M., Franceschini, C., Wallerstein, N., Mercer, R., Cimmino, K., Rodriguez, L., Latinovic, L., Pliego, P., Rodriguez, J. O. V., Aguillón, G. L. P., Groot, A. M. M., Monteiro, T., Rivière-Cinnamond, A., Franco de Sá, R., \& Hegel, G. (2017). Healthy Municipalities, Cities, and Comunities in Latin America: Strong Stories, Commited Futures. In E. De Leeuw \& J. Simos (orgs.). Healthy Cities - The Theory, Policy, and Practice of Value-Based Urban Planning (pp. 151-214). New York: Springer.

Sperandio, A. M. G. (org.) (2003). o processo de construção da Rede de Municípios Potencialmente Saudáveis (Vol. 1). Campinas: Unicamp.

Sperandio, A. M. G. (org.) (2004a). O processo de construção da Rede de Municípios Potencialmente Saudáveis. (Vol. 2). Campinas: IPES Editorial.

Sperandio, A.M.G.(org.). (2004b). O processo de construção da Rede de Municípios Potencialmente Saudáveis - Repensando a mobilização e a participação social. Volume 3. Campinas: Unicamp.

Sperandio, A. M. G. \& Vilarta, R. (orgs.) (2004). Tecendo a Sustentabilidade da Rede de Municípios Potencialmente Saudáveis - Diferentes abordagens da mobilização social (Vol. 4). Campinas: IPES.

Sperandio, A. M. G. \& Serrano, M. M. (orgs.) (2006). O Plano Diretor: uma ferramenta para o desenvolvimento das políticas públicas de um município potencialmente saudável (Vol. 1). Campinas: Unicamp \& Brasília: OPAS.

Wilding, H., Gould, R., Taylor, J., Sabouraud A., Saraux-Salaun, P., Papathanasopoulou, D., de Blasio, A., Nagy, Z., \& Simos, J. (2017). Healthy Cities in Europe: Structured, Unique and Thoughtful. In E. De Leeuw \& J. Simos (orgs.). Healthy Cities - The Theory, Policy, and Practice of Value-Based Urban Planning (pp. 241-292). New York: Springer. 